which was associated with toothache. A decayed tooth was removed, and the hearing completely restored. Two teeth have since been removed under similar circum. stances and with precisely similar results.

Mr. Tomes remarked, in the discussion upon the paper, that "it seemed a rational conclusion that the deafness was the immediate result of the diseased teeth, inas. much as that condition occurred on three separate occasions, and the operation was attended in each case with the same results." (British Journal of Dental Science, Feb. 1863.)

My object in contributing to this journal cases of dental pathology, is to show how essential it is in all cases of facial neuralgia, etc., that the mouth should be carefully examined, although, as I have before stated, no teeth may be actually painful, yet by their presence in a diseased condition they may be acting as the sole cause of the mischief. It is the province of the dentist to carry out his profession upon the principles of conservatism ; but even this treatment may be carried too far.

" How far," asks Mr. Catlin, " should dental conservative surgery be carried? To preserve teeth as long as they can be made subservient to the uses of mastication and appearance is undoubtedly the first duty of the dentist ; but when they have become sufficiently diseased to affect the general health, or so painful as to interrupt the ordinary duties and comfort of life, the prevalent conservative dental surgery of the present day becomes mis chievous." (British Journal of Dental Science, February 18(53.)

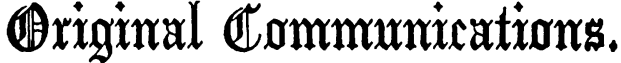

\section{ADDITIONAL NOTE ON THE OCCURRENCE OF MALIGNANT PUSTULE IN ENGLAND.}

By Wiruram Budd, M.D., Clifton.

IN the paper on Malignant Pustule which $I$ had the honour to read at the last annual meeting of the Asso. ciation, I ventured on the opinion that the reason why this disease has remained hitherto almost unnoticed in England is, not that it does not occur, but that, except by one or two observers, its real nature has not been recognised.

In confirmation of this opinion I may state that, during and since the publication of the paper in the JounNaL, I have been favoured by medical men living in various parts of England with numerous histories of characteristic cases of this peculiar and striking malady. Two of the number, on account of their special interest, may be fitly recorded here, by way of appendix to those related in the paper itself.

For my knowledge of the first of the two, I am indebted to the kindness! of Mr. Nunneley of Leeds. The data which Mr. Nunneley has placed in my hands consist of two drawings with memoranda attached. One of the drawings represents the head of the patient. The appearances are in the highest degree characteristic. It is very remarkable that, although the disease began in the hand, the head and face present precisely the same aspect as that which is observed when it begins in these parts. There are the same enormous swelling, the same black discoloration, and, in particular, the same thickening and negro.like protuberance of the lips, which were such striking features of the cases which came under my own observation, and in all of which the disease began in the mouth or in the immediate neigh. hood of it.

The subject of Mr. Nunneley's case was a young man,
19 years old, and a plasterer by trade. His illness occurred in April 1856. About a month before, he was bitten on the knuckle of the left forefinger by a young dog. The dog was quite well. The wound, which was small, festered, and was still open at the time of death. It did not, however, prevent the man from continuing his work. The disease began in the injured hand.

"When I first saw him," Mr. Nunneley says, "the arm was enormously swollen and greatly discoloured, as were also the face and head." These last parts were nearly twice their natural size.

The whole of the body and limbs, but more particu. larly the lower limbs, were covered with purple blotches. When these first appeared, they were of a brownish-red; but they afterwards became violet, and showed a ten. dency to spread. In some of these blotches, vesicles containing a dark-coloured serum formed around a central patch of a somewhat lighter shade. (These appearances are represented in the second drawing.)

The patient died six days after the onset of the malady. The mind continued unimpaired to the last.

On examination of the body, the lungs were found much congested, and there was dark coloured serum in both pleuræ. The blood was diffluent.

This case occurred at the time when the poisoning cases of Dove and Palmer were so much canvassed by the public, and it was in consequence of Mr. Nunneley's connection with them that he was called in to the patient. The fact is worth recording, as showing two things: first, that the disease was of a very uncommon character; and secondly, that the friends of the patient associated the idea of poisoning with it.

Mr. Nunneley himself says : "The appearance of the young man was so peculiar that I never saw anything like it before"; and several other eminent surgeons who visited the patient with him made the same remark.

The aspect of the case, as exhibited in the drawing, was equally strange to the medical gentlemen who gave evidence for the defence on Palmer's trial, and not one of the number could form even a guess as to the cause of the malady.

Mr. Nunneley rightly supposed that it was a case of " the malignant pustule of the continent."

A clue to its probable origin, apparently overlooked by these gentlemen, seems to me to be furnished by the calling of the patient. Two points in the history may be taken to be pretty sure. The first is, that the man was not inoculated with malignant pustule by the dog; the second, that, as the disease began in the injured part, its virus must have been subsequently received through the open wound. To complete the chain of evidence, we must remember that, in spite of the sore on his finger, the patient continued to work as usual. Now, plasterers are constantly handling bullock's hair, which is a large ingredient of mortar; and as bullock's hair is known to be a very common vehicle of the charbon virus, it seems to me to be highly probable that the wounded finger received the contagion from this source.

For the particulars of the second case, I am indebted to the kindness of Dr. Milner Barry of Tunbridge Wells; and $I$ give them in his own words:-

"Mr. J. E., aged 47, a respectable butcher of this town, was apparently in perfect health on the morning of Sunday, July 14th, 1861. In the evening, as he was sitting with his family, he was observed to be picking at a little sore on his upper lip, a little below his right nostril. He had only then noticed its presence, having had his attention directed to the lip by a peculiar itching and tingling sensation, which compelled him to keep rubbing the sore spot.

"On Monday, July 15th, there was no change, and he did not complain of feeling ill.

"On the 16th, he went to Tunbridge market, a distance of five miles; and having returned home early, he went to the hay-field to see how his haymaking was get- 
ting on. Thinking that his haymakers were working rather sluggishly, he scolded them for their idleness, threw off his coat, and set to work lustily, to show them a good example. It was a warm and rather moist day, the temperature in the shade being $73^{\circ}$. One of the men who was working next to Mr. E. remarked that he did not seem to get hot, or to perspire, from bis exertions, although the sweat was pouring down his own face profusely. In the afternoon, Mr. E. felt ill, and went home and lay down, but was well enough to come down to tea. The lip continued to be itchy, and to tease him, but did not swell. He allayed the itching by bathing it repeatedly with warm water. He had a restless, uncomfortable night; but got up early next morning (July 17), and went off to the hay-field. After working there vigorously for an hour, he was compelled to give up and return home; and immediately went to his bedroom and called to his wife to help him to bed, for he felt very ill. When in bed, he said that he should never leave that bed again, and he proceeded to give directions respecting his affairs. His usual medical attendant was sent for, and noticed that he looked pale, and that there was apparently a little gathering in the lip.

"On Thursday morning, July 18th, the swelling of the lip had increased, and appeared to contain matter. It was lanced, but only a little reddish serum was discharged from the wound. The swelling increased rapidly during the day, and spread over the face; and by evening, the right eye was closed.

"On Friday, July 19, the lip was considerably swollen, and the swelling and discoloration extended completely over the face. The left eye was closed.

“ On Saturday morning, July $20 \mathrm{th}$, at ten o'clock, I saw him for the first time, in consultation with his regular medical attendant, an experienced surgeon.

"Mr. E. had been a handsome, well.featured man. He was now so frightfully disfigured that $I$ could not re. cognise him. The face seemed featureless, swollen out of all shape and comeliness, and of a livid, purple hue. The upper lip was frightfully enlarged; the eyes were closed tightly; the eyelids were puffy, discoloured, and cdematous, and their edges crusted over with a gummy exudation. The swelling and discoloration affected the whole of the face, and all the anterior region of the head. He was propped up in bed, muttering deliriously; his hands trembling, his breathing hurried, the skin perspiring, the belly tympanitic, and the pulse so feeble and so frequent that it could not be accurately counted.

"Brandy and stimulants were freely administered; but he continued to sink, and he died about three hours after my first visit. The disease ran its course in seven days."

Three other cases, of whieh the particulars have been communicated to me, may be mentioned here as interesting in relation to the question of cause. The subject of one was a tanner; of another a shepherd, who had been engaged in tending diseased sheep; while a third was seized with malignant pustule in the hand immediately after being employed in unloading hides. In all three the disease was fatal. In the five cases which form the subject of this note, therefore, the circum. stances not only pointed strongly to an animal origin, but brought the sufferers into actual contact with things which are recognised as common sources of the malady of which they died.

Of the cases related in the body of the paper, there were two in which the contagion was implanted by flies (a well known mode of communication), and one in which it was derived from contact with the carcase of a sheep that had died in a field. The callings of four other subjects were equally suggestive, one being a bullock jobber in a large way, another a great flock mas. ter, a third a provision dealer, and a fourth a shepherd. That is to say, in twelve out of thirty cases, without any inquiry being once directed to the point, the disease was ascertained to occur, under the circumstances, or in the conditions of life, in which the malignant pustule of the continert is known most to happen.

As it is impossible to suppose this to have been the work of chance, the only rational explanation of the fact is that these conditions and circumstances operated in developing malignant pustule in these twelve persons merely by exposing them to the specific cause of the malady. Had the real nature and origin of the affection been suspected by the observer, contact with this cause would probably have been actually traced in many of these as well as in other instances.

As regards the probable intervention of flies in the propagation of the disease, it is worthy of mention that, with the exception of one case which occurred in the latter end of March, all the cases of malignant pustule of the face that have come to my knowledge have hap. pened either in the summer or early autumn, and for the most part in dry, hot weather. The cases in which it has occurred in the hands (presumably from direct contact with the virus) have happened at various seasons of the year.

Lastly, it should be remarked that in the five cases which form the subject of this note, as in the twentyfive before related, the disease occurred in the only parts of the body which are habitually uncovered.

\section{PUERPERAL ECLAMPSIA : BRIGHT'S DISEASE : AUTOPSY.}

By Paur Belcher, Esq., Burton-on-Trent.

ON January Ist, 1863, I was sent for to see Mrs. C. (a multipara), who was expecting her confinement daily; and had just had an epileptic fit. She had been taken quite suddenly; the fit had lasted about a quarter of an hour; and she was now recovering. The power of vision was quite lost; her pupils were natural but sluggish. She had violent pain in the head with throbbing. She had passed very little urine to-day.

She was ordered ten grains of calomel immediately; some jalap mixture every four hours; and twelve leeches to the bead immediately.

The first fit was at about ten o'clock. At four o'clock, she had a second fit lasting a quarter of an hour; and was bled from the arm to faintness, losing about twelve ounces. At nine o'clock, she had a third fit, lasting twenty minutes. Shortly afterwards she had a turpentine enema, which acted well. Her head was ordered to be shaved, and cold bladders were applied. Her pulse was now 110, and she expressed herself as better, and had recovered ber sight. The blood was highly buffed and cupped. Her urine was scanty, and highly albuminous. There were no signs of labour.

Jan. 2nd. She had had some sleep. Pulse 108. She complained of her tongue, which was much bitten. Her urine was scanty, and brandy-coloured. She was ordered to repeat the calomel at bedtime, and to have added to the mixture bitartrate of potash in half-drachm and spirits of nitrous ether in drachm doses.

Jan. 3rd. She had slept well. The bowels acted well, and she passed rather more urine, which was still highly albuminous, and showed casts of various sizes, some granular, others containing atrophied and fatty epithelium. Her pulse was 100. She was perfectly free from labour-pain, and thought she had a week to go.

Jau. 4th. She had slept well. The pulse was more thrilling and somewhat quicker. She complained of uneasiness as if labour were going to supervene. At 4 P.Mr, she was seized with another fit, lasting a quarter of an hour, followed by coma for a few minutes, and then by another paroxysm, again succeeded by coma. She was immediately bled to fainting, losing fifteen ounces in a 